

\title{
Evaluation of the Normal Cochlear Second Interscalar Ridge Angle and Depth on 3D T2-Weighted Images: A Tool for the Diagnosis of Scala Communis and Incomplete Partition Type II
}

\author{
(D)T.N. Booth, (D) C. Wick, (D) R. Clarke, (D).W. Kutz, DM. Medina, DD. Gorsage, (D) Y. Xi, and (D) B. Isaacson
}

\begin{abstract}
BACKGROUND AND PURPOSE: Cochlear malformations may be be subtle on imaging studies. The purpose of this study was to evaluate the angle and depth of the lateral second interscalar ridge or notch in ears without sensorineural hearing loss (normal ears) and compare them with ears that have a documented incomplete type II partition malformation.
\end{abstract}

MATERIALS AND METHODS: The second interscalar ridge notch angle and depth were measured on MR imaging in normal ears by a single experienced neuroradiologist. The images of normal and incomplete partition II malformation ears were then randomly mixed for 2 novice evaluators to measure both the second interscalar ridge notch angle and depth in a blinded manner. For the mixed group, interobserver agreement was calculated, normal and abnormal ear measurements were compared, and receiver operating characteristic curves were generated.

RESULTS: The 94 normal ears had a mean second interscalar ridge angle of $80.86^{\circ} \pm 11.4^{\circ}$ and depth of $0.54 \pm 0.14 \mathrm{~mm}$ with the 98 th percentile for an angle of $101^{\circ}$ and a depth of $0.3 \mathrm{~mm}$. In the mixed group, agreement between the 2 readers was excellent, with significant differences for angle and depth found between normal and incomplete partition type II ears for angle and depth on average ( $P<.001)$. Receiver operating characteristic cutoffs for delineating normal from abnormal ears were similar for both readers (depth, 0.31/0.34 mm; angle, $\left.114^{\circ} / 104^{\circ}\right)$.

CONCLUSIONS: A measured angle of $>114^{\circ}$ and a depth of the second interscalar ridge notch of $\leq 0.31$ mm suggest the diagnosis of incomplete partition type II malformation and scala communis. These measurements can be accurately made by novice readers.

ABBREVIATIONS: IP-II = incomplete partition type II; R2 = second interscalar ridge; SNHL = sensorineural hearing loss

S ensorineural hearing loss (SNHL) can occur due to congenital or acquired conditions. Congenital malformations of the cochlea are generally reported with a descriptive classification using the number of turns and do not specifically include some of the subtler cochlear anomalies. One of the most frequently encountered abnormalities in patients with congenital malformations of the inner ear is enlargement of the endolymphatic duct. ${ }^{1,2}$ Enlargement or increased diameter of the en-

Received September 21, 2017; accepted after revision January 12, 2018.

From the Departments of Radiology (T.N.B., R.C., D.G.) and Otolaryngology (C.W., J.W.K., M.M., B.I.), Children's Medical Center of Dallas, University of Texas Southwestern Medical Center, Dallas, Texas; and Department of Radiology (Y.X.), University of Texas Southwestern Medical Center, Dallas, Texas.

Paper previously presented at: Annual Meeting of the American Society of Neuroradiology and the Foundation of the ASNR Symposium, April 22-27, 2017; Long Beach, California.

Please address correspondence to Timothy N. Booth, MD, Department of Radiology, Children's Medical Center of Dallas, 1935 Medical District Dr, Dallas, TX 75235; e-mail: Tim.booth@children.com; @tbooth278

http://dx.doi.org/10.3174/ajnr.A5585 dolymphatic duct is commonly associated the cochlear anomalies with a classic association of incomplete partition type II (IP-II) malformation. This cochlear malformation may be difficult to diagnose, and more objective criteria are needed for accurate assessment.

Anatomically, the continuous osseous spiral lamina separates the cochlea into 2 visible perilymph-containing chambers on MR imaging, the scala tympani and scala vestibuli, with the intervening endolymph-containing scala media not currently demonstrable on imaging. Imaging may demonstrate scalar defects and/or scalar asymmetry with enlargement of the scala vestibuli, which may be seen in association with cochlear malformations. While scala communis can be associated with scalar asymmetry, it is a more extensive malformation that results from a defect of the osseous interscalar septum, which separates the cochlear turns. This is a continuous structure that spans the entire length of the cochlea and results in a continuous ridge along the outer margin, separating it into 3 distinct turns, resulting in 3 distinct indentations or ridges along the 


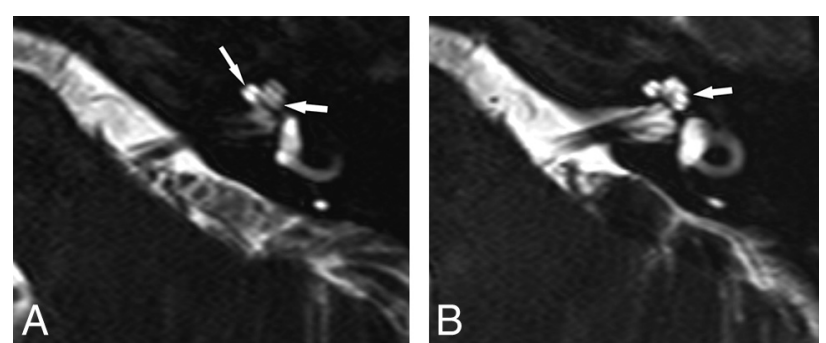

FIG 1. Axial 3D T2-weighted images from inferior to superior showing a normal osseous spiral lamina and interscalar septum. A, A normal osseous spiral lamina separating the scala tympani (posterior) and scala vestibuli (anterior) of the basal (anterior arrow) and middle turns (posterior arrow). B, A normal lateral R2 notch (arrow) associated with the second part of the interscalar septum between the upper basal and upper middle turns of the cochlea.

margins of the cochlea. The second interscalar ridge (R2) or notch, associated with the interscalar septum and located along the lateral margin of the cochlea, is most easily appreciated on imaging (Fig 1).,

Scala communis is a common developmental malformation of the cochlea, which manifests as incomplete formation of the distal interscalar septum with resultant incomplete separation of the cochlear turns. This abnormality can be isolated (normal number of cochlear turns) or associated with incomplete partition defects, most commonly type II. ${ }^{3,5}$ The presence of cochlear malformation can result in more intraoperative challenges at the time of and after a cochlear implant operation, with decreased speech perception (outcome) and a risk for a CSF gusher. ${ }^{6}$ A CSF gusher typically occurs secondary to a deficient modiolus and lamina cribrosa, whereas a perilymphatic gusher is usually seen in the presence of an enlarged endolymphatic duct and sac. Appropriate classification of cochlear malformations is important for presurgical planning and in predicting outcomes. ${ }^{7}$ A thorough knowledge of cochlear anatomy and experience in interpreting MR imaging studies is requisite in the diagnosis and classification of inner ear malformations because imaging findings are often subtle.

Using axial 3D T2-weighted MR imaging, one can easily visualize the R2 notch formed by the second portion of the interscalar septum in normal ears; therefore, it is hypothesized that a decrease in depth or increase in the angle of this notch is associated with cochlear scalar malformations. The purpose of this study was to analyze and compare the R2 depth and angle in a group of children with normal hearing (no SNHL) with a subset of patients with ears that have an IP-II malformation. A secondary objective was to determine the feasibility of measuring the R2 angle and depth by comparing the measurements of 2 novice readers.

\section{MATERIALS AND METHODS}

This was a retrospective case-control study. The study was approved by the institutional review board and was Health Insurance Portability and Accountability Act-compliant. Axial TSE 3D T2-weighted driven equilibrium MR imaging (TR, 3000 ms; TE, $230 \mathrm{~ms}$; NEX, 1; slice thickness, $1.5 \mathrm{~mm}$ at $0.75 \mathrm{~mm}$; matrix, $419 \times$ 364) was performed through the temporal bone as part of a routine cholesteatoma protocol. We retrospectively searched the electronic medical record for this examination code. These patients served as our healthy cohort. Patients were excluded if a 3D sequence was not present or if the sequence was determined to be of low quality (nonvisualization of the osseous spiral lamina), an audiogram was not available for review, SNHL was present, or the patient was diagnosed with a syndrome known to be associated with SNHL. All audiograms were reviewed by a neurotology fellow (C.W.) for the presence of SNHL defined as a $>20-\mathrm{dB}$ mean bone line. Radiology reports and a clinical data base were used to identify patients with an IP-II malformation. The images were reviewed, and the diagnosis was confirmed by an experienced pediatric neuroradiologist and neurotologist (T.N.B., B.I.). If available, genetic results were reviewed for the patients with an IP-II malformation.

In the healthy cohort, the R2 angle and depth measurements were performed using electronic calipers on a PACS station by a pediatric neuroradiologist with 20 years of experience (T.N.B.). The images were magnified 2-3 times to adequately measure the area of interest. A measurement of $<0.2 \mathrm{~mm}$ was considered zero because electronic calipers would not generate measurement below this value, and the maximum angle was $180^{\circ}$. Additionally, the images from the healthy cohort were evaluated for additional abnormalities of the cochlea, vestibule, and semicircular canals, including visualization of the second portion of the interscalar septum, any partition defects, modiolar deficiency, and scalar asymmetry and/or defects and for the presence or absence of the cochlear nerve. The vestibular labyrinth was evaluated with specific attention to the semicircular canals and the size of the bone island between the vestibule and lateral semicircular canal as well as the size of the endolymphatic duct. An endolymphatic duct midaperture diameter of $\geq 1 \mathrm{~mm}$ was considered abnormal.

A mixed cohort of ears was constructed that included normal ears randomly extracted from the healthy cohort $(n=23)$ and ears with a confirmed IP-II malformation $(n=17)$. The ears were randomly and blindly presented to a pediatric radiology fellow (D.G.) and a third-year otolaryngology resident (M.M.) to measure both the R2 angle and depth. There were a total of 40 ears in the mixed cohort. Three of the IP-II malformation cases were unilateral with normal hearing on the contralateral side. Because readers had limited experience in interpreting MR imaging studies of the temporal bone, they reviewed a reference article ${ }^{3}$ and completed 5 cases on a normal-hearing ear before measuring the study scans. The blinded readers were told to only evaluate the R2 notch, and cases were presented in 2 sessions with unilateral healthy cases presented to decrease bias.

In the healthy cohort of patients evaluated by the single experienced reader, analysis included determination of the range of measurements of the normal-hearing R2 angle and depth as well as calculating the $98 \%$ measurements, upper for angle and lower for depth. Using a mixed cohort of normal-hearing and IP-II malformed ears, we evaluated novice reader agreement by the intraclass correlation coefficient. The average of the 2 readers was used for further analysis. A linear mixed model was used to determine the difference in mean angle and depth between normal and abnormal ears. Interpatient correlation was considered a random effect in the linear model and was subsequently evaluated by the Wald $Z$-test. A receiver operating characteristic curve was also used to assess the diagnostic performance of angle and depth measurements in detecting abnormalities. The corresponding area under the receiver operating characteristic curve was calculated 

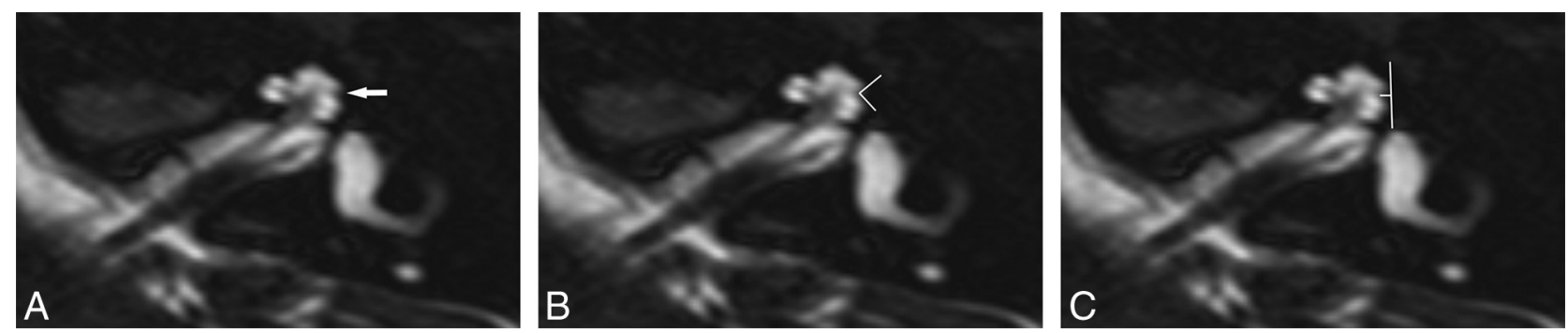

FIG 2. Axial 3D T2-weighted image through the cochlea in a normal-hearing ear. A, A normal lateral notch is demonstrated (arrow). $B$ and $C, A t$ the same level, angle and depth measurements are demonstrated using electronic calipers (lines). The angle was measured as $84^{\circ}$ with a depth of $0.6 \mathrm{~mm}$.
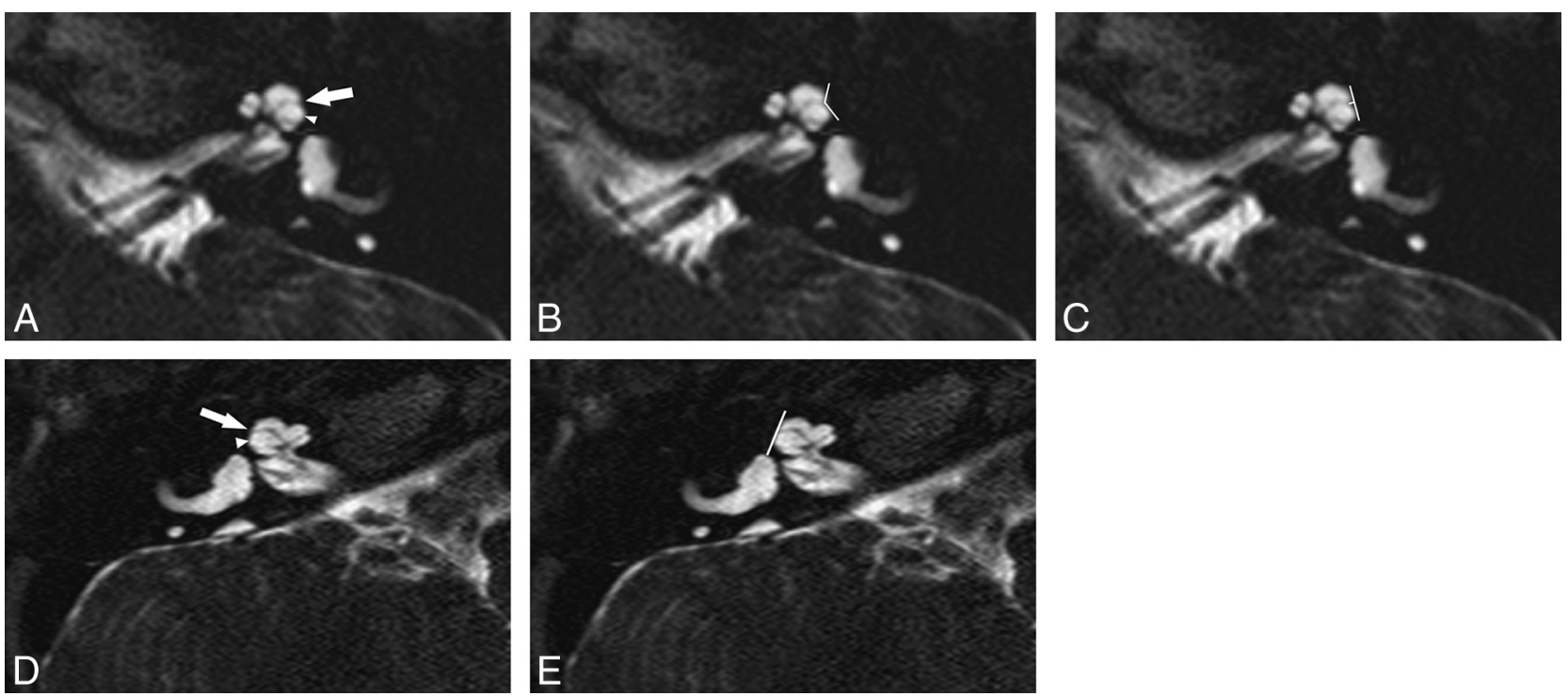

FIG 3. Abnormal R2 notch in 2 different IP-II malformation ears, both with profound SNHL. A-C, A shallow obtuse notch is present (arrow). The scala vestibuli is enlarged (arrowheads) with anterior bowing and high insertion of the second part of the interscalar septum. The measured angle at the same level is $132^{\circ}(B)$ with a depth of $0.3 \mathrm{~mm}(C)$. $D$ and $E$, In the other patient, no lateral notch is seen (arrow) and the scala vestibuli is enlarged (arrowhead). At the same level, the angle is $180^{\circ}$ and the depth is $0 \mathrm{~mm}(E)$.

together with $95 \%$ confidence intervals. $P<.05$ was considered statistically significant. The optimal cutoff was determined by the Youden J statistic. The corresponding sensitivity and specificity were also calculated. SAS 9.4 (SAS Institute, Cary, North Carolina) was used for all analyses.

\section{RESULTS}

\section{Healthy Cohort}

Sixty-nine patients or 138 ears had a 3D T2-weighted MR imaging performed that was considered of good quality. We excluded the following patients: Fourteen patients (28 ears) did not have an audiogram available, and 1 patient (2 ears) had branchio-otorenal syndrome. After we evaluated the audiogram, 14 ears had SNHL and were excluded, resulting in 94 ears ( 52 patients) in the healthy cohort. Thirty-four patients were male and 18 were female, with an age at imaging range of 2-17 years (mean,10.3 years). Most patients were imaged on a $1.5 \mathrm{~T}$ magnet $(n=51)$.

The 94 normal ears had a mean R2 angle of $80.9^{\circ} \pm 11.4^{\circ}$ (range, $57^{\circ}-132^{\circ}$ ) and a depth of $0.54 \pm 0.14 \mathrm{~mm}$ (range, $0.3-0.9$ $\mathrm{mm}$ ). The 98 th percentile angle was $101^{\circ}$ with a depth of $0.31 \mathrm{~mm}$ (Fig 2). There was no significant difference in any measurement with age $(P=.8)$ or sex $(P=.37)$. Morphologic analysis of the membranous labyrinth demonstrated a normal cochlea in all nor- mal ears with normal hearing. The second portion of the interscalar septum was seen in 34 ears $(36 \%)$. Cochlear nerves were present in all ears. Five ears (5\%) showed a diminutive bony island separating the lateral semicircular canal from the vestibule. No patient had a dilated endolymphatic duct.

\section{Mixed Cohort}

Seventeen ears with IP-II malformation were confirmed. Seven patients were male, and 3 were female with an age range of $0.8-6$ years (mean, 2.9 years) (Fig 3). As per consensus evaluation, a displaced second portion of the interscalar septum was present in 15 ears with associated scalar asymmetry. The second portion of the interscalar septum was not identified in 2 ears. Vestibular abnormalities were present in 8 ears, typically enlargement of the vestibule with diminished size of the bone island in 8 ears and endolymphatic duct enlargement in 12 ears. The bony island was normal and the endolymphatic duct was not enlarged in any of the unilateral IP-II malformation ears $(n=3)$. The cochlear nerve was normal in all ears. Genetic testing was performed in only 2 patients. Connexin was normal in 1 patient, and 1 patient was heterozygous for SLC26A4 with 2 separate disease-causing mutations.

The mixed group comprising 17 ears with IP-II malformation was combined with 23 ears from the normal-hearing group, in- 


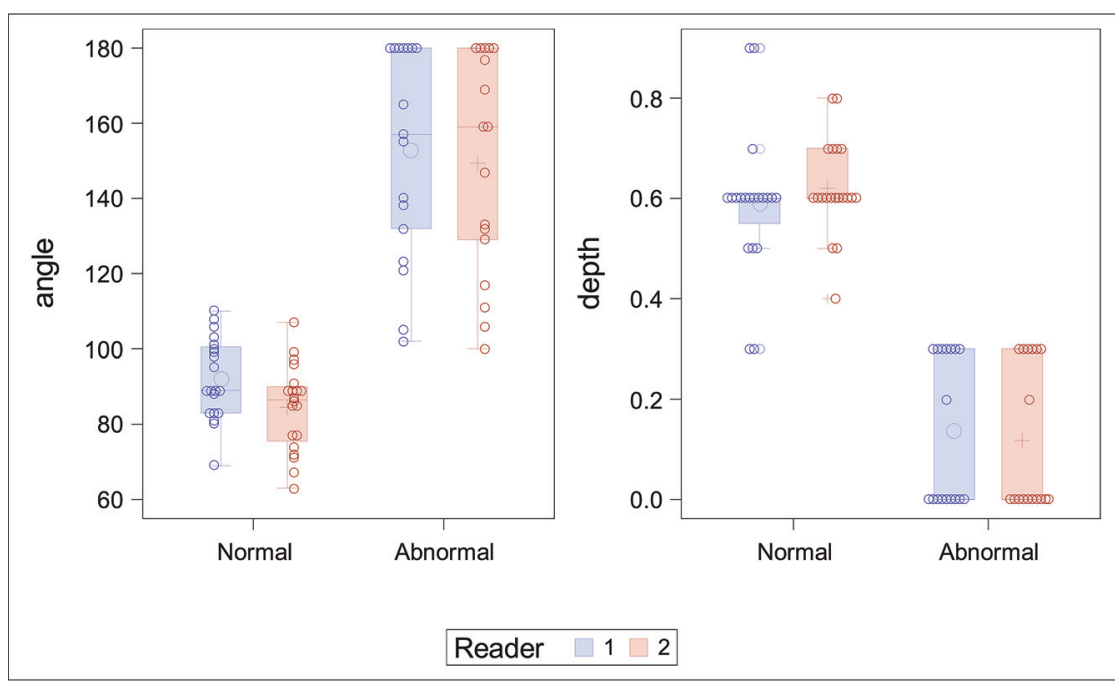

FIG 4. Distribution of measurements of normal and abnormal ears by both novice readers.

ROC analysis for the 2 novice readers

\begin{tabular}{lccccc}
\hline ROC Model & AUC & $\begin{array}{c}95 \% \text { Confidence } \\
\text { Interval }\end{array}$ & $\begin{array}{c}\text { Optimal } \\
\text { Cutoff }\end{array}$ & Sensitivity & Specificity \\
\hline Reader 1 & & & & & \\
$\quad$ Angle & 0.9676 & $0.9177-1$ & 114.156 & $88 \%$ & $100 \%$ \\
$\quad$ Depth (mm) & 0.9588 & $0.8983-1$ & 0.31294 & $100 \%$ & $90 \%$ \\
Reader 2 & & & & & \\
$\quad$ Angle & 0.9853 & $0.9593-1$ & 103.874 & $94 \%$ & $95 \%$ \\
$\quad$ Depth $(\mathrm{mm})$ & 0.9647 & $0.8953-1$ & 0.33536 & $100 \%$ & $95 \%$ \\
\hline
\end{tabular}

Note:- ROC indicates receiver operating characteristic; AUC, area under the curve.

cluding 3 contralateral normal-hearing ears from the patients with unilateral IP-II malformation. The age range was $1-10$ years (mean, 5 years), and the sex distribution of 8 males and 5 females of the normal-hearing group was like that in the IP-II group. In this mixed cohort, the agreement between the 2 novice reader measurements was excellent (intraclass correlation coefficient for angle, 0.8 ; 95\% CI, 0.65-0.89; intraclass correlation coefficient for depth, 0.73 ; 95\% CI, $0.54-0.85)$. Angle was not significantly different either by age $(P=.27)$ or $\operatorname{sex}(P=.62)$. However, younger children had significantly shallower R2 depth $(P=.001)$ but not $\operatorname{sex}(P=.46)$. A significant difference was found between normalhearing and IP-II malformation ears for angle $(P<.001)$ and depth $(P<.001)$ (Fig 4$)$. Receiver operating characteristic analysis suggested an excellent area under curve using angle or depth, independent of the observer. Optimal cutoffs for angle and depth were similar between the 2 readers, with high sensitivity and specificity (Table).

\section{DISCUSSION}

Inner ear malformations are found in $20 \%$ of patients with congenital SNHL. ${ }^{8}$ Cochlear malformations have been subdivided into categories, with cochlear aplasia representing the most severe end of the spectrum and IP-II malformation representing a milder malformation. ${ }^{1}$ MR imaging is commonly performed in the evaluation of children with SNHL due to the ability to directly visualize the cochlear nerve. ${ }^{9}$ Appropriate classification of cochlear malformations on MR imaging is clinically important to assess the prognosis for cochlear implantation and for potential complications. $^{6}$
Embryologically, the interscalar septum forms after the formation of the perilymphatic spaces. Membranous bony struts extend toward the modiolus to form the interscalar septum, which separates the turns of the cochlea. The interscalar septum is a continuous structure that can be divided into 3 parts, which form 3 ridges or notches (R1, R2, R3) along the outer margin of the cochlea. The second and third parts of the interscalar septum, which are abnormal in IP-II malformations, form via membranous ossification with the first part of the septum, forming via enchondral ossification. ${ }^{4,10}$ The second part of the interscalar septum divides the upper basal and upper middle turns (lateral), and the third part divides the lower middle from the apical turn (medial). The first part of the interscalar septum divides the lower basal from the lower middle turn (posterior medial). The R2 notch is formed by the associated second part of the interscalar septum and is located along the lateral margin of the cochlea. In our experience, this notch is well-demonstrated on high-resolution MR imaging.

Other cochlear findings such as scalar asymmetry with enlargement of the scala vestibuli, defects in the osseous spiral lamina, and enlargement of the endolymphatic duct are typically seen in IP-II malformation and were commonly present in our IP-II cases. Reported vestibular abnormalities, including decreased size of the lateral semicircular canal bone island with enlargement of the lateral semicircular canal, were found in our affected ears as well. ${ }^{11}$ The vestibule and semicircular canals were normal in the unilateral cases of IP-II malformation, and dilated endolymphatic ducts were not present. This malformation likely would have been classified as an IP-IIa by one author with associated milder involvement of the cochlea. ${ }^{12}$ These ears may benefit even more from a thorough evaluation of the $\mathrm{R} 2$ notch because the cochlear abnormality may be subtler and there is a lack of confirmatory vestibular abnormalities.

The second part of the interscalar septum was only visualized in $36 \%$ of the normal-hearing ears and appears more difficult to evaluate than the associated notch on MR imaging using our technique. The septum can also be difficult to distinguish from the adjacent osseous spiral lamina in healthy patients, and visualization may be limited due to resolution. Thus, the presence or absence was not evaluated in the mixed-cohort group.

Most interesting, the interscalar septum in IP-II malformations is visualized on histopathology; however, it is often not seen on CT because of a higher insertion point and lack of ossification. ${ }^{4,12}$ Evaluation of the R2 notch on CT would be of value and further research into normal and abnormal values is needed. In IP-II malformation ears, the second portion of the septum bulges anteriorly and is associated with an enlarged scala vestibule, which was commonly found 
in our cases (Fig 4). ${ }^{13}$ In a recent study, the second part of the interscalar septum was routinely identified using a higher resolution T2 drive sequence at $3 \mathrm{~T}$. The authors proposed evaluating the distance between the osseous spiral lamina of the upper basal turn and the first signal void anteriorly, presumably the second part of the interscalar septum. A cutoff value of $\geq 1.2 \mathrm{~mm}$ was found useful for diagnosing the IP-II malformation. ${ }^{14}$

The normal R2 notch along the lateral margin of the cochlea was easily appreciated on high-resolution T2-weighted images in the healthy cohort of ears. Identifying abnormalities of the R2 notch may be helpful in the evaluation of children with SNHL, and it has been reported to be abnormal on histologic examination as well as in a few imaging reports. ${ }^{3,4}$ In patients with normal hearing, the notch was measurable in all cochleas, with the 98th percentile for angle and depth being $101^{\circ}$ and $0.3 \mathrm{~mm}$, respectively, by an experienced reader.

Using a mixed group of normal ears and ears with an imagingconfirmed diagnosis of IP-II malformation, novice readers had excellent agreement in evaluating the angle and depth of the R2 notch. A significant difference in measurements was found when comparing normal ears with ears with an IP-II malformation, as well as similar cutoffs for angle and depth using receiver operating characteristic analysis $\left(0.31 / .34 \mathrm{~mm}\right.$ and $114^{\circ} / 104^{\circ}$, respectively). An R2 notch measuring $>114^{\circ}$ or $\leq 0.31 \mathrm{~mm}$ is suggested as evidence of an IP-II malformation or scala communis. There was a correlation with having a shallower notch with younger age in both groups, maybe due to an imaging-resolution issue or possibly related to postnatal maturation of the interscalar septum. The ability of novice readers to accurately evaluate the R2 notch is important because these examinations can be difficult to interpret and evaluation of the lateral notch is a simple reproducible method to evaluate the integrity of the R2 septum and therefore the presence or absence of scala communis.

Two of the 10 patients with IP-II underwent genetic testing, one of which was for connexin 26 only, which had normal findings, and the remaining patient had a compound heterozygous mutation in the SLC26A4 gene. The SLC26A4 gene codes for the protein pendrin, which is a chloride/bicarbonate anion exchanger that serves to increase the $\mathrm{pH}$ in the endolymph compartment by secreting bicarbonate. ${ }^{15}$ Pendrin protein expression in the endolymphatic sac is critical in the development of the mammalian inner ear as demonstrated in a murine model. The absence or reduction in pendrin expression results in acidification and abnormal expansion of the developing inner ear. Acidification of the developing inner ear results in reduced endolymphatic potassium concentration and subsequent loss of the endocochlear potential. An increase in endolymph calcium also results from acidification and thus results in toxicity to the stria vascularis and the organ of Corti. $^{15}$

Potential limitations of the study include bias in the interpretation of the healthy cohort because the experienced reader did have a preconceived theory of the appearance of the normal R2 notch. Also, while the inexperienced readers had minimal prior exposure to interpreting MR imaging of the temporal bone, other findings encountered in IP-II malformation may have been seen and affected their interpretation.

\section{CONCLUSIONS}

The R2 notch, formed by the associated second part of the interscalar septum, should be evaluated in all patients being imaged with a diagnosis of SNHL. This notch is easily appreciated with reproducible measurements on high-resolution MR imaging. A $\mathrm{R} 2$ angle of $>114^{\circ}$ and/or a depth of $\leq 0.31 \mathrm{~mm}$ considered abnormal. Measurement of the R2 notch may aid in the evaluation of more subtle IP-II malformations or an isolated scala communis.

Disclosures: Joe W. Kutz-UNRELATED: Consultancy: Medtronic, Purdue Pharma, Achaogen. Brandon Isaacson—UNRELATED: Board Membership: Med-EL, Advanced Bionics, Comments: Advisory Board member; Consultancy: Advanced Bionics, Medtronic, Stryker Corporation, Karl Stortz SE \& Company KG, Olympus Corporation, Comments: electrode testing, drill testing, and designing an endoscopic ear surgery tool and imaging development.

\section{REFERENCES}

1. Sennaroglu L, Saatci I. A new classification for cochleovestibular malformations. Laryngoscope 2002;112:2230-41 CrossRef Medline

2. Adibelli ZH, Isayeva L, Koc AM, et al. The new classification system for inner ear malformations: the INCAV system. Acta Oto Laryngol 2017;137:246-52 CrossRef Medline

3. Makary C, Shin J, Caruso P, et al. A histological study of scala communis with radiological implications. Audiol Neurotol 2010;15: 383-93 CrossRef Medline

4. Leung KJ, Quesnel AM, Juliano AF, et al. Correlation of CT, MR, and histopathology in incomplete partition-II cochlear anomaly. Otol Neurotol 2016;37:434-37 CrossRef Medline

5. Merchant SN. Genetically determined and other developmental defects. In: Merchant SN, Nadol JB, eds. Schuknecht's Pathology of the Ear. 3rd ed. New York: McGraw-Hill Medical; 2010:545-611

6. Sennaroglu L, Sarac S, Ergin T. Surgical results of cochlear implantation in malformed cochlea. Otol Neurotol 2006;27:615-23 CrossRef Medline

7. Papsin BC. Cochlear implantation in children with anomalous cochleovestibular anatomy. Laryngoscope 2005;115(1 Pt 2 Suppl 106): 1-26 Medline

8. Sennaroglu L. Cochlear implantation in inner ear malformation: a review article. Cochlear Implants Int 2010;11:4-41 CrossRef Medline

9. Glastonbury CM, Davidson $\mathrm{CH}$, Harnsberger HR, et al. Imaging findings of cochlear nerve deficiency. AJNR Am J Neuroradiol 2002; 23:635-43 Medline

10. Som PM, Curtin HD, Liu K, et al. Current embryology of the temporal bone, part I: the inner ear. Neurographics 2016;6:250-65 CrossRef

11. Davidson CH, Harnsberger HR, Lemmerling MM, et al. MR evaluation of vestibulocochlear anomalies associated with large endolymphatic duct and sac. AJNR Am J Neuroradiol 1999;20:1435-41 Medline

12. Kontorinis G, Goetz F, Giourgas, et al. Radiologic diagnosis of incomplete partition type I versus type II: significance for cochlear implantation. Eur Radiol 2012;22:525-32 CrossRef Medline

13. Sennaroglu L. Histopathology of inner ear malformations: do we have enough evidence to explain pathophysiology? Cochlear Implants Int 2016;17:3-20 CrossRef Medline

14. Reinshagen KL, Curtin HD, Quesnel AM, et al. Measurement for detection of incomplete partition type II anomalies on MR imaging. AJNR Am J Neuroradiol 2017;38:2003-07 CrossRef Medline

15. Wangemann P. Mouse model for pendrin-associated loss of cochlear and vestibular function. Cell Physiol Biochem 2013;32(Suppl 1):157-65 CrossRef Medline 